\title{
Prazosin protects myocardial cells against anoxia-reoxygenation injury via the extracellular signal-regulated kinase signaling pathway
}

\author{
LIXIN WANG ${ }^{*}$, YAN XUE* , HAO MA, HAIYAN SHI, LING WANG and XIAOZHENG CUI \\ Department of Cardiovascular Surgery, The General Hospital of Chinese People's Armed Police Force, \\ Beijing 100039, P.R. China
}

Received October 8, 2016; Accepted July 11, 2017

DOI: $10.3892 / \mathrm{mmr} .2017 .8175$

\begin{abstract}
Ischemic heart disease (including coronary arterial atherosclerosis, or vascular cavity stenosis or occlusion) remains the leading cause of disease-associated mortality worldwide. Prazosin, a receptor blocker of postsynaptic adrenaline, is essential in expanding peripheral arteries, which decreases peripheral vascular resistance, and regulates anti-hypertensive action. However, the mechanisms underlying the effects of prazosin have not been fully elucidated. The aim of the present study was to investigate the protective effects of prazosin on myocardial cells against anoxia-reoxygenation injury in a mouse model. The regulatory effects of prazosin on blood lipid levels and blood pressure were investigated in experimental mice. Furthermore, inflammation responses and oxidative stress in myocardial cells were analyzed in mice treated with prazosin. Apoptotic myocardial cells were investigated in experimental mice treated with prazosin. In addition, apoptotic gene expression levels were evaluated in myocardial cells. Extracellular signal-regulated kinase (ERK) expression and phosphorylation was investigated in myocardial cells in mice with anoxia-reoxygenation injury following prazosin treatment. The activity and expression levels of nuclear factor of activated T cells (NF-AT), activator protein 1 (AP-1) and necrosis factor $(\mathrm{NF})-\kappa \mathrm{B}$ were observed in myocardial cells. Furthermore, histological analyses were performed to investigate the benefits of prazosin treatment on anoxia-reoxygenation injury. The results of the present study identified that prazosin decreased the expression levels of inflammatory factors, interleukin (IL)-6, tumor necrosis factor
\end{abstract}

Correspondence to: Professor Lixin Wang, Department of Cardiovascular Surgery, The General Hospital of Chinese People's Armed Police Force, 69 Yongding Road, Beijing 100039, P.R. China E-mail: wanglixinprof@163.com

${ }^{*}$ Contributed equally

Key words: prazosin, anoxia-reoxygenation injury, apoptosis, inflammation, oxidative stress, extracellular signal-regulated kinase
(TNF)- $\alpha$, IL-10 and IL-1 in the serum of mice exhibiting hypoxia/reoxygenation injury. Oxidative stress was observed to be improved and the apoptosis rate was decreased in myocardial cells in anoxia-reoxygenation injury model mice treated with prazosin. ERK expression and phosphorylation was upregulated, and expression levels of NF-AT, AP-1 and $\mathrm{NF}-\kappa \mathrm{B}$ were downregulated in the myocardial cells of mice treated with prazosin. Blood lipid levels and blood pressure of the anoxia-reoxygenation injury model mice were markedly improved following treatment with prazosin. Histological analysis indicated that the area, circumference fragmentation and segmentation of myocardial cells were significantly improved following prazosin treatment. Thus, these results indicate that prazosin treatment decreases inflammation responses, oxidative stress, and apoptosis of myocardial cells via regulation of the ERK signaling pathway. The findings indicate that prazosin may present as a potential therapeutic agent for the treatment of hypoxia/reoxygenation injury.

\section{Introduction}

Cardiovascular disease is a generic term, incorporating cardiovascular and cerebrovascular diseases caused by hyperlipidemia, sticky blood, atherosclerosis, and hypertension $(1,2)$. Cardiovascular disease presents systemic vascular lesions affecting the performance of the heart and brain, and is the most frequent cause of mortality in the adult population of economically developed countries $(3,4)$. Coronary heart disease is a type of disease that includes coronary atherosclerosis heart disease, coronary arterial atherosclerosis and vascular cavity stenosis or occlusion, which cause myocardial ischemia, hypoxia or necrosis of the heart $(5,6)$. Anoxia-reoxygenation injury of the heart is a serious threat to human health and coronary heart disease, caused by myocardial ischemia-reperfusion injury, is the primary reason for mortality and disability during cardiovascular disease, which emerges during myocardial infarction, cardiopulmonary bypass surgery, heart attack, and heart transplantation, and during other types of cardiovascular disease $(7,8)$. Anoxia-reoxygenation injury of heart frequently and ultimately results in irreversible fatal injury and even mortality (9). Therefore, elucidating the molecular 
mechanism of anoxia-reoxygenation injury is considered to be essential for the prevention and treatment of cardiovascular disease.

Inflammation is one of the most common characteristics of anoxia-reoxygenation injury of the heart (10). A previous study indicated that preventing inflammation efficiently protects against myocardial ischemia-reperfusion injury (11). Vinten-Johansen et al (12) demonstrated that inflammation and pro-inflammatory mediators are involved in myocardial ischemia-reperfusion injury, post-ischemic injury and gradually restore blood flow. In addition, a previous study indicated that inhibition of apoptosis and inflammation contributes to rehabilitation of myocardial ischemia/reperfusion injury (13). Furthermore, a previous study demonstrated the beneficial role of prazosin on cardiovascular manifestations and pulmonary edema (14). However, to the best of our knowledge, the efficacy of prazosin on inflammation has not been investigated. In the present study, changes in serum inflammatory factors were analyzed in mice with anoxia-reoxygenation injury following treatment with prazosin.

Oxidative stress is another inducer of anoxia-reoxygenation injury of the heart and may lead to dyslipidemia, impairment of energy metabolism and chronic kidney disease (15). Matsuda et al (16) indicated that anti-oxidative agents improve insulin resistance and restore adiponectin production in obesity-associated metabolic and cardiovascular diseases (16). In addition, oxidative stress in cardiovascular disease has demonstrated important implications in physiological and pathophysiological cardiovascular processes (17). Furthermore, oxidative stress and antioxidant strategies in cardiovascular disease and cardiovascular disease-associated parameters have been investigated in a model for metabolic syndrome $(18,19)$. Furthermore, the long-term effects of prazosin administration on blood pressure, heart and structure of coronary artery have been evaluated in young spontaneously hypertensive rats (20). In the present study, the efficacy of prazosin on oxidative stress was analyzed in a mouse model of anoxia-reoxygenation injury. Notably, the molecular mechanism of prazosin on anoxia-reoxygenation injury was investigated in experimental mice.

The aim of the present study was to investigate the protective effects of prazosin on myocardial cells against anoxia-reoxygenation injury in a mouse model. The regulatory effects of prazosin on inflammation responses, oxidative stress, blood lipid levels, blood pressure and apoptosis were analyzed in experimental mice. The potential mechanism of ERK-mediated nuclear factor of activated T cells (NF-AT), AP-1, NF- $\kappa$ B signaling pathways was also investigated in myocardial cells in mice with anoxia-reoxygenation injury following prazosin treatment. These investigations indicate that the long-term effect of prazosin is beneficial for improvement of anoxia-reoxygenation injury.

\section{Materials and methods}

Ethical approval. The current study was performed according to the recommendations of the Guide for the Care and Use of Laboratory Animals of the General Hospital of Chinese People's Armed Police Force (Beijing, China). All surgery and experiments were performed under anesthetic to minimize pain.
Cell cultures. Myocardial cells from experimental mice were cultured in Minimum Essential Medium (Gibco; Thermo Fisher Scientific, Inc., Waltham, MA, USA) with $10 \%$ fetal bovine serum (Gibco; Thermo Fisher Scientific, Inc.) and incubated overnight at $37^{\circ} \mathrm{C}$ in a humidified atmosphere of $5 \%$ $\mathrm{CO}_{2}$. Myocardial cells were treated with $10 \mathrm{mg} / \mathrm{ml}$ prazosin (P7791; Sigma-Aldrich; Merck KGaA, Darmstadt, Germany) for $24 \mathrm{~h}$ to analyze the therapeutic effects of prazosin in vitro.

ELISA. In the protein detection assay, mouse IL-6 (D6050), TNF- $\alpha$, (MTA00B) IL-10 (DY417), IL-1 (MLB00C), interferon (IFN) $-\gamma$ (DY485) and IL-2 (M2000; all R\&D Systems, Inc., Minneapolis, MN, USA). ELISA kits were used to determine the serum levels of inflammatory factors. The procedures were conducted according to the manufacturer's instructions. The final results were recorded at a wavelength of $450 \mathrm{~nm}$ using an ELISA plate reader.

Western blot analysis. Myocardial cells were homogenized in lysate buffer containing protease-inhibitor (P8340; Sigma-Aldrich; Merck $\mathrm{KGaA}$ ) and were centrifuged at $8,000 \times \mathrm{g}$ at $4^{\circ} \mathrm{C}$ for $10 \mathrm{~min}$. The supernatant was used for analysis of protein expression. For detection of purpose protein, transmembrane proteins were extracted using a Transmembrane Protein Extraction kit (Qiagen Sciences, Inc., Gaithersburg, MD, USA) according to the manufacturer's instructions. Protein concentration was measured using a bicinchoninic acid assay kit (Thermo Fisher Scientific, Inc.). Protein samples $(20 \mu \mathrm{g})$ were separated by $12 \%$ SDS-PAGE and transferred onto PVDF membranes (EMD Millipore, Billerica, MA, USA) as previously described (21). For western blotting, primary antibodies against the following were used: Reactive oxygen species (ROS; 1:1,000; ab151922), glutathione (GSH; 1:1,000; ab94733), heme oxygenase (HO)-1 (1:1,000; ab68477), P38 mitogen activated protein kinase (MAPK; 1:1,000; ab197348), catalase (CAT; 1:1,000; ab52477), superoxide dismutase (SOD; 1:1,000; ab80946), Caspase-3 (1:1,000; ab13847), Caspase-9 (1:1,000; ab202068), B-cell lymphoma (Bcl)-2 (1:1,000; ab692), P53 (1:1,000; ab1431), apoptotic protease activating factor 1 (Apaf-1; 1:1,000; ab2001), Fas (1:1,000; ab82419), ERK (1:1,000; ab54230), pERK (1:1,000; ab65142), NF-AT (1:1,000; ab25916), AP-1 (1:1,000; ab21981), NF-кB (1:1,000; ab32360) and $\beta$-actin (1:1,000; ab5262; all Abcam, Cambridge, UK) for $12 \mathrm{~h}$ at $4^{\circ} \mathrm{C}$ following blocking with $5 \%$ skimmed milk for $1 \mathrm{~h}$ at $37^{\circ} \mathrm{C}$. Membranes were subsequently incubated with horseradish peroxidase-conjugated goat anti-rabbit $\operatorname{IgG} \mathrm{mAb}(1: 2,000$; PV-6001; OriGene Technologies, Inc., Beijing, China) for $24 \mathrm{~h}$ at $4^{\circ} \mathrm{C}$. A Ventana Benchmark automated staining system was used to analyze protein expression (Olympus BX51; Olympus Corp., Tokyo, Japan).

Flow cytometric analysis of myocardial cell apoptosis. Apoptosis rates of myocardial cells were evaluated using Annexin V-fluorescein isothiocyanate (FITC) and propidium iodide (PI) apoptosis detection kit (556547; BD Biosciences, San Jose, CA, USA). Myocardial cells were collected and suspended with Annexin V-FITC and PI according to the manufacturer's instructions. Fluorescence was measured via FACS scan flow cytometry (BD CellQuest Software Version 3.3; BD Biosciences). 

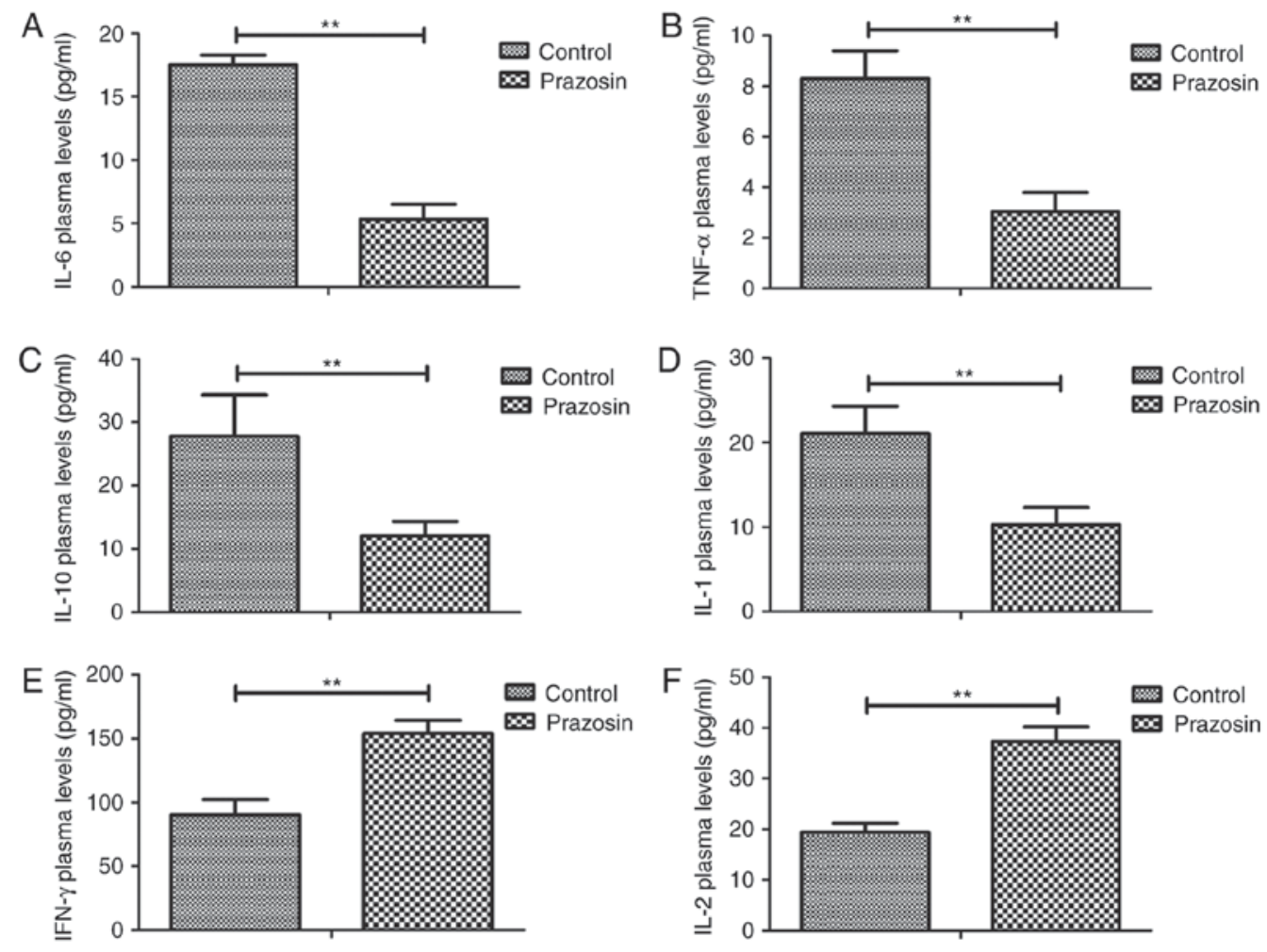

Figure 1. Effects of prazosin on serum expression levels of inflammatory factors in mouse models of anoxia-reoxygenation injury. Inflammatory factor expression levels of (A) IL-6, (B) TNF- $\alpha$, (C) IL-10 and (D) IL-1 in the serum of mouse models of anoxia-reoxygenation injury. Plasma concentration levels of (E) IFN- $\gamma$ and (F) IL-2 in mouse models of anoxia-reoxygenation injury. The results are presented as means and standard deviation of three independent experiments. ${ }^{*} \mathrm{P}<0.05,{ }^{* *} \mathrm{P}<0.01$ vs. control group. IL, interleukin; TNF, tumor necrosis factor; IFN, interferon.

Animal experiments. A total of 120 male C57BL/6 mice were purchased from Charles River Laboratories (Shanghai, China) and housed under pathogen-free conditions. Mice were maintained under a $12 \mathrm{~h}$ light/dark cycle with free access to food and water. The myocardial injury mouse model was established according to a previous study (22). Following establishment of the anoxia-reoxygenation injury mouse model, the mice were randomized into two groups ( $\mathrm{n}=60$ per group). The animals received prazosin $(10 \mathrm{mg} / \mathrm{kg})$ treatment or the same volume of phosphate-buffered saline (PBS) once per day and the treatment continued for 30 days. Cardiac function was further analyzed to evaluate the efficacy of prazosin.

Immunohistochemistry. The myocardial tissues were flash frozen at $-20^{\circ} \mathrm{C}$ and cut into sections $(4 \mu \mathrm{m})$, deparaffinized in xylene and rehydrated through a graded ethanol series. Sections were prepared and epitope retrieval was performed using a Lab Vision ${ }^{\mathrm{TM}}$ Tris-HCl Buffer for Heat-Induced Epitope Retrieval kit (AP9005; Invitrogen; Thermo Fisher Scientific, Inc.) for further analysis. The paraffin sections were subjected to hydrogen peroxide (3\%) for 10-15 min and subsequently blocked using $5 \%$ skim milk for $10-15 \mathrm{~min}$ at $37^{\circ} \mathrm{C}$. Finally, the sections were stained with hematoxylin and eosin at $4^{\circ} \mathrm{C}$ for $12 \mathrm{~h}$. All sections were washed three times with PBS. The area of myocardial injury, circumference fragmentation and segmentation of myocardial cells were determined by observation of six randomly selected views under a fluorescence microscope.

Statistical analysis. All data are presented as means and standard error of the mean. Unpaired data was analyzed using Student's t-test. Comparison of data between multiple groups was performed by one-way analysis of variance and $\mathrm{P}<0.05$ was considered to indicate a statistically significant difference.

\section{Results}

Prazosin decreases serum levels of inflammatory factors in the mouse model of anoxia-reoxygenation injury. Expression levels of inflammatory factors were analyzed in the anoxia-reoxygenation injury mouse model prior to and post treatment with prazosin, as determined by ELISA. As shown in Fig. 1A-D, inflammatory factor expression levels of IL- 6 , TNF- $\alpha$, IL-10 and IL-1 were decreased by prazosin treatment in the serum of mice with hypoxia/reoxygenation injury. However, plasma concentration levels of IFN $-\gamma$ and IL-2 were significantly upregulated by prazosin in mice with hypoxia/reoxygenation injury (Fig. 1E, F). These findings indicate that prazosin decreased the levels of inflammatory factors in the serum and promoted secretion of anti-inflammatory factors in the mouse model of anoxia-reoxygenation injury.

Prazosin downregulates oxidative stress in the mouse model of anoxia-reoxygenation injury. To investigate the biological effects of prazosin on myocardial cells, oxidative stress was analyzed in the myocardial cells of mice with anoxia-reoxygenation injury. As shown in Fig. 2A-D, ROS, GSH, p38MAPK and CAT were markedly downregulated in myocardial cells in a prazosin-treated mouse model of anoxia-reoxygenation injury. However, the expression levels of SOD and HO-1 
A
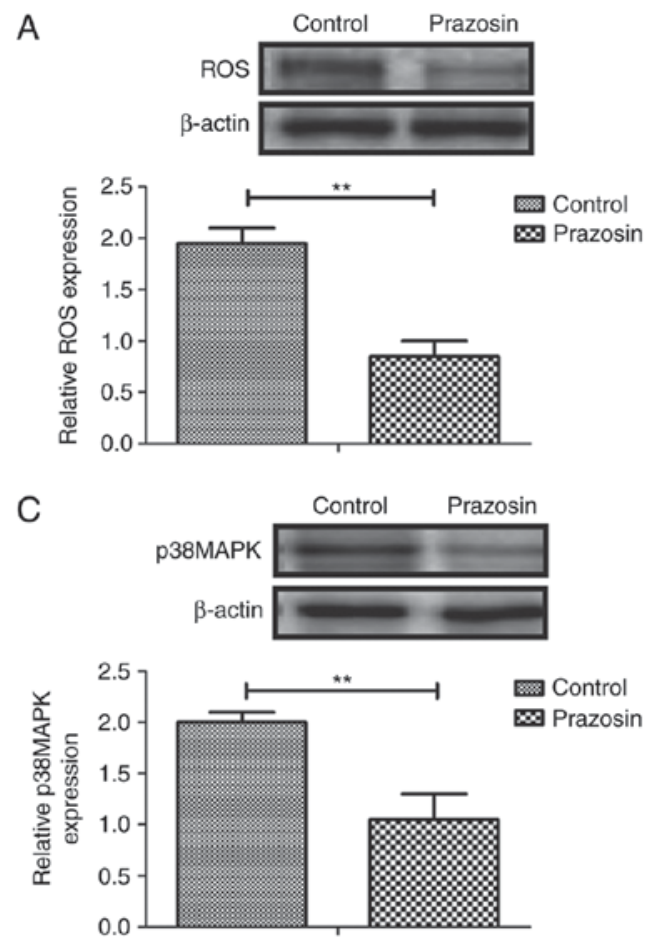

E

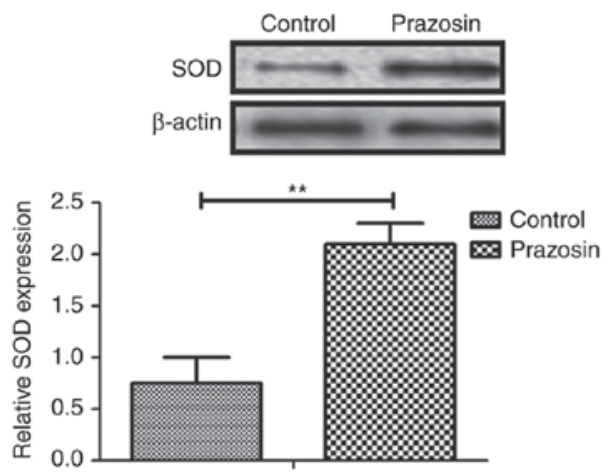

B

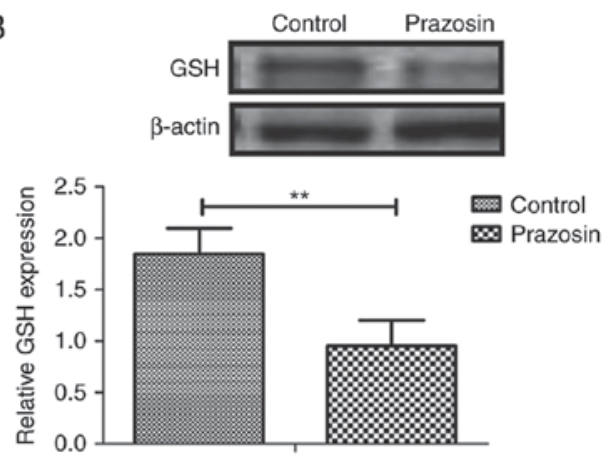

D

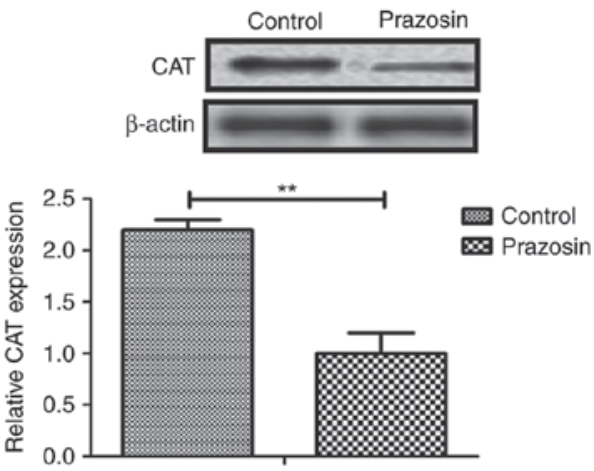

$\mathrm{F}$

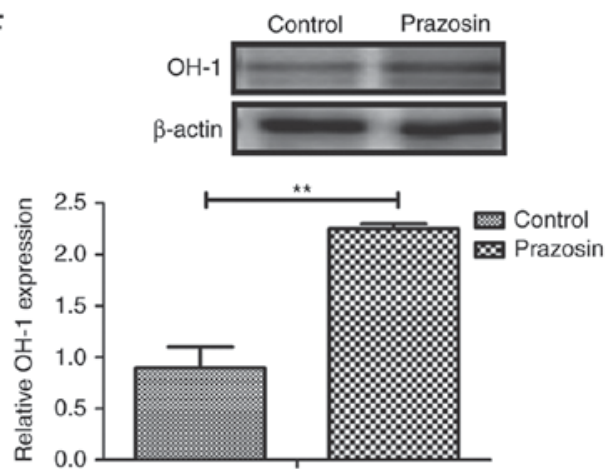

Figure 2. Effects of prazosin treatment on oxidative stress a in a mouse model of anoxia-reoxygenation injury. Expression levels of (A) ROS, (B) GSH, (C) p38 MAPK and (D) CAT in myocardial cells in a mouse model of anoxia-reoxygenation injury. Protein expression levels of (E) SOD and (F) HO-1 in the prazosin-treated mouse models of anoxia-reoxygenation injury compared with the control. The results are presented as means and standard deviation of three independent experiments. ${ }^{*} \mathrm{P}<0.05,{ }^{* * *} \mathrm{P}<0.01$ vs. control group. ROS, reactive oxygen species; GSH, glutathione; MAPK, mitogen-activated protein kinases; CAT, catalase; SOD, superoxide dismutase; HO-1, heme oxygenase-1.

were upregulated in the myocardial cells of prazosin-treated mice with anoxia-reoxygenation injury (Fig. 2E, F). These results indicate that prazosin downregulates oxidative stress and upregulates SOD and HO-1 activation in mice exhibiting anoxia-reoxygenation injury.

Prazosin suppresses apoptosis of myocardial cells in a mouse model of anoxia-reoxygenation injury. Subsequently, the improvement of apoptosis in myocardial cells was analyzed in the mouse models of anoxia-reoxygenation injury following treatment with prazosin. As demonstrated in Fig. 3A, the apoptosis rate was decreased in the experimental mice treated with prazosin. In addition, pro-apoptotic gene expression levels of caspase- 3 and caspase- 9 expression were observed to be downregulated following prazosin $(10 \mathrm{mg} / \mathrm{ml})$ treatment (Fig. 3B). Furthermore, expression levels of B-cell lymphoma 2 and $\mathrm{p} 53$ were upregulated in the myocardial cells of the mouse models of anoxia-reoxygenation injury following treatment with Prazosin (Fig. 3C). Furthermore, following treatment with prazosin, the expression levels of Apaf-1 and Fas were downregulated in the myocardial cells of the mouse models of anoxia-reoxygenation injury (Fig. 3D). There results indicate that prazosin contributes to the protection of prazosin against apoptosis in myocardial cells.

Prazosin improves survival of myocardial cells in mice with anoxia-reoxygenation injury via the ERK signaling pathway. The growth and molecular mechanism of prazosin-mediated improvement of myocardial cells were further analyzed in mice with anoxia-reoxygenation injury. As presented in Fig. 4A, growth of myocardial cells was promoted by prazosin in the experimental mice. The survival rate of myocardial cells was improved by prazosin treatment in mice with anoxia-reoxygenation injury (Fig. 4B). In addition, the expression and phosphorylation levels of ERK were observed to be stimulated 
A
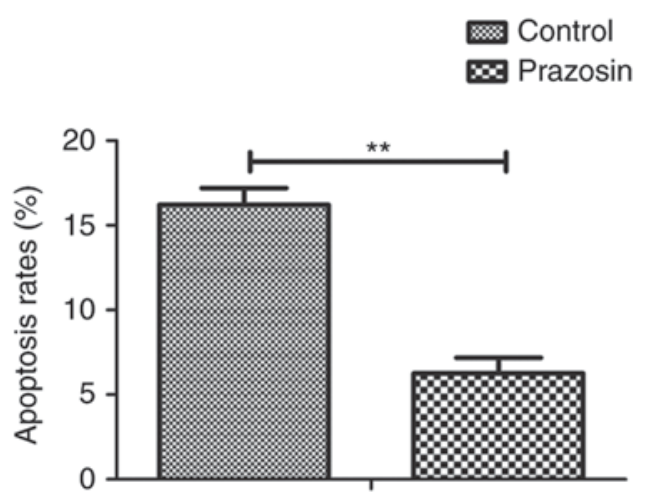

C
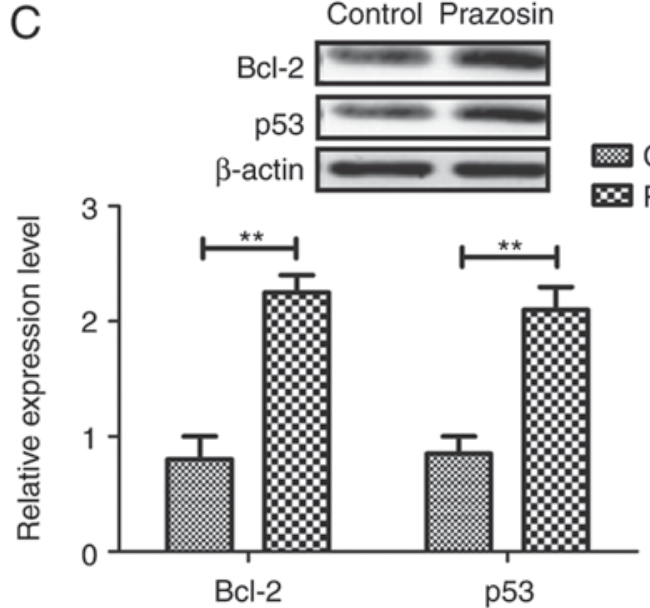

B
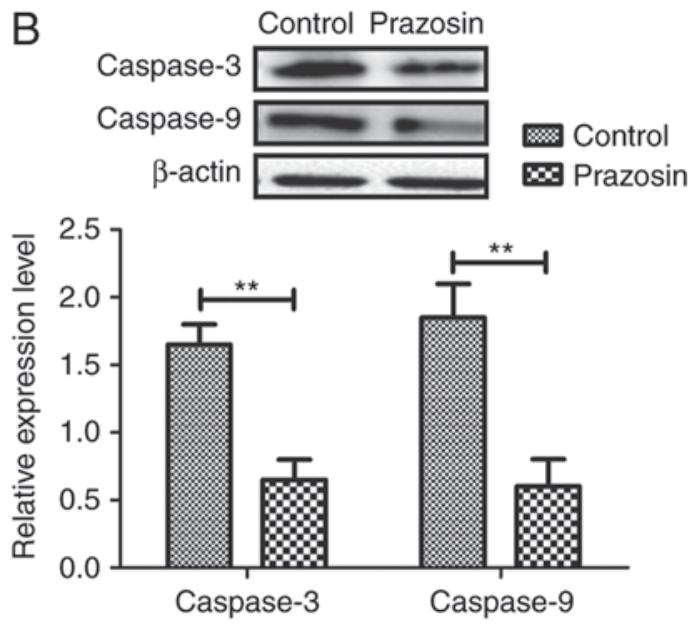

Control Prazosin
D
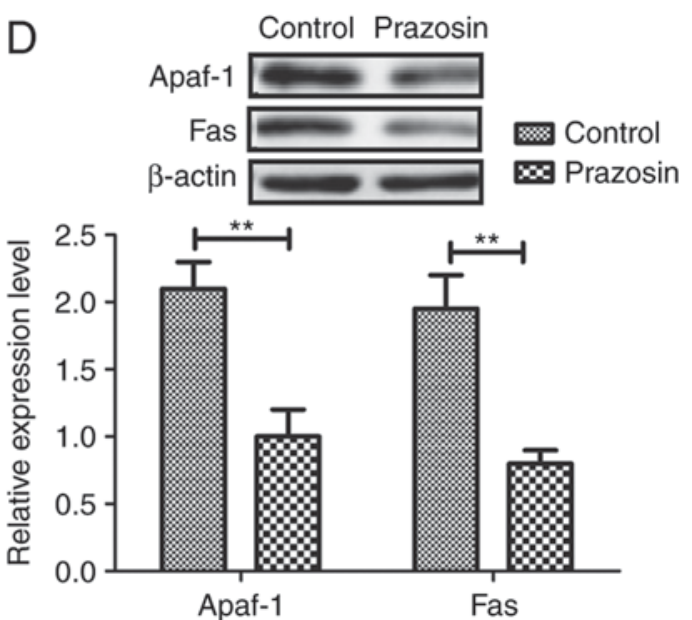

Figure 3. Effects of prazosin on apoptosis of myocardial cells in a mouse model of anoxia-reoxygenation injury. (A) Apoptosis rate of myocardial cells, (B) pro-apoptosis gene expression levels of caspase-3 and caspase-9, and (C) expression levels of Bcl-2 and p53 in the myocardial cells of the prazosin-treated mouse models of anoxia-reoxygenation injury compared with the control. (D) Expression levels of Apaf-1 and Fas were downregulated in the myocardial cells of mice with anoxia-reoxygenation injury following treatment with prazosin or phosphate-buffered saline. The results are presented as the means and standard deviation of three independent experiments. ${ }^{*} \mathrm{P}<0.05,{ }^{* *} \mathrm{P}<0.01$ vs. control group. Bcl-2, B-cell lymphoma 2 ; Apaf-1, apoptotic protease activating factor 1.
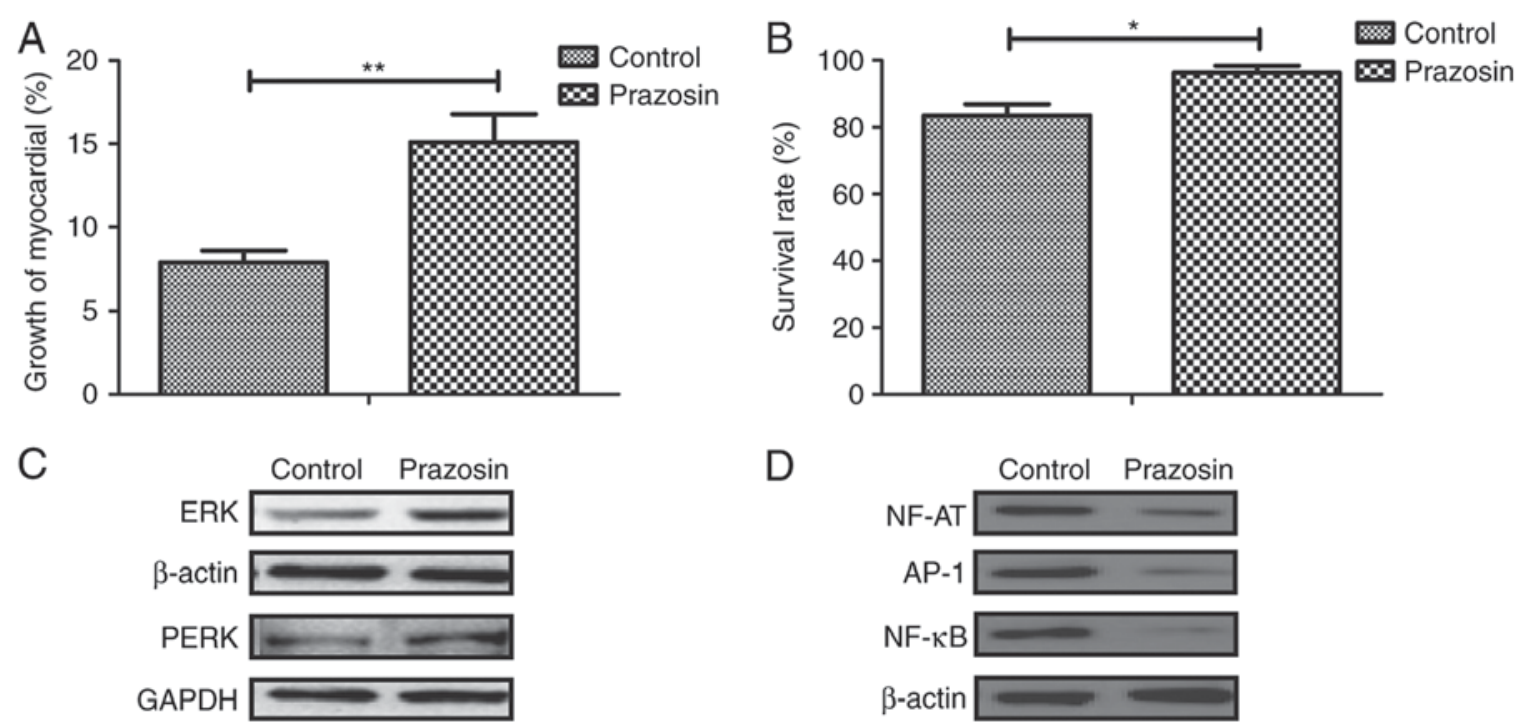

Figure 4. Effects of prazosin on survival of myocardial cells in mice with anoxia-reoxygenation injury via the ERK signaling pathway. (A) Growth and (B) survival rate of myocardial cells in prazosin-treated mice with anoxia-reoxygenation injury compared with the control. (C) Expression and phosphorylation levels of ERK in myocardial cells in the experimental mice. (D) Expression levels of NF-AT, AP-1 and NF-кB in myocardial cells in mice treated with prazosin or phosphate-buffered saline. The results are expressed as means and standard deviation of three independent experiments. " $\mathrm{P}<0.05$, ${ }^{* *} \mathrm{P}<0.01$ vs. control group. ERK, extracellular-signal regulated kinase; NF-AT, nuclear factor of activated T cells; AP-1, activator protein 1; NF- $\mathrm{kB}$, nuclear factor- $\mathrm{kB}$. 
A

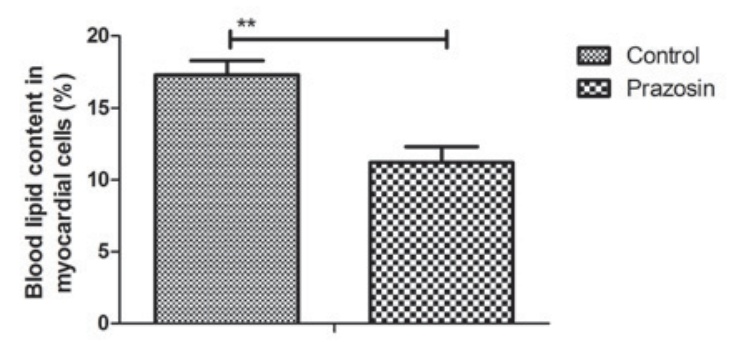

C

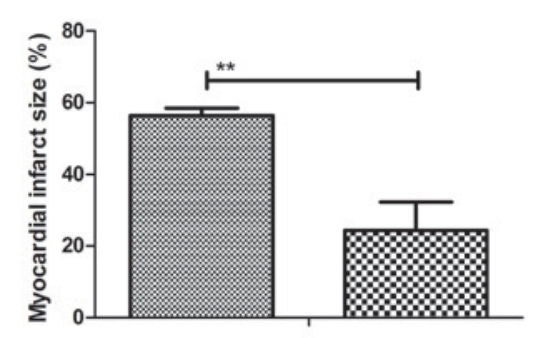

B

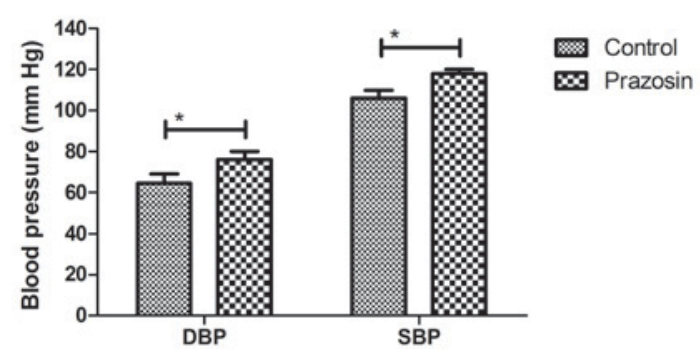

D

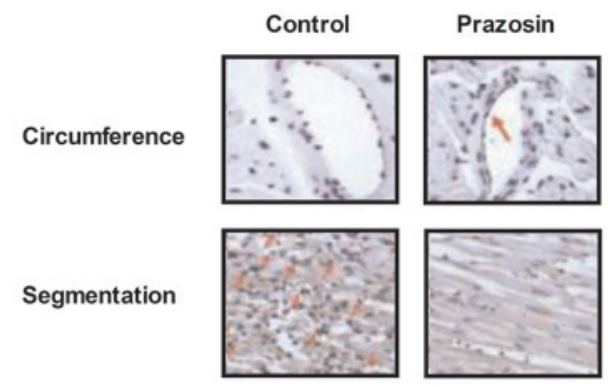

Figure 5. Effects of prazosin on blood lipid levels, blood pressure, and myocardial function in mice with anoxia-reoxygenation injury. (A) Content of blood lipids in myocardial cells in mice treated with prazosin or phosphate-buffered saline. (B) Blood pressure of mice following prazosin treatment in mice with anoxia-reoxygenation injury compared with the control. (C) Area of myocardial injury, and (D) Circumference fragmentation and segmentation of myocardial cells in the prazosin-treated mice with anoxia-reoxygenation injury compared with the control. Magnification, $\mathrm{x} 40$. Arrow indicates the circumference fragmentation. The results are expressed as the means and standard deviation of three independent experiments. ${ }^{*} \mathrm{P}<0.05,{ }^{* *} \mathrm{P}<0.01 \mathrm{vs}$. control group.

by prazosin in experimental mice (Fig. 4C). Furthermore, the expression levels of NF-AT, AP-1, NF- $\mathrm{B}$ were downregulated in myocardial cells in mice treated with prazosin (Fig. 4D). These results indicate that prazosin improves survival in the myocardial cells of mice with anoxia-reoxygenation injury via the ERK signaling pathway.

Prazosin improves blood lipid levels, blood pressure and myocardial function in mice with anoxia-reoxygenation injury. Myocardial function was analyzed in experimental mice following treatment with prazosin. As presented in Fig. 5A, blood lipid levels were improved in a prazosin-treated mouse model of anoxia-reoxygenation injury. In addition, blood pressure was improved following prazosin treatment in the experimental mice (Fig. 5B). In addition, the area of myocardial injury was markedly decreased in the myocardial tissue of mice treated with prazosin (Fig. 5C). Furthermore, circumference fragmentation and segmentation of myocardial cells were markedly improved by prazosin treatment, as determined by histological analysis (Fig. 5D). These results indicate that prazosin improves blood lipid levels, blood pressure, and myocardial function in mice with anoxia-reoxygenation injury.

\section{Discussion}

Coronary heart disease remains one of biggest causes of death in the world and is closely associates with metabolic disorders of endogenous substances (23).
Anoxia-reoxygenation injury is a serious type of coronary heart disease that significantly promotes apoptosis of myocardial cells, and increases inflammation and oxidative stress $(24,25)$. In the current study, the efficacy of prazosin against anoxia-reoxygenation injury in a mouse model was investigated. The results demonstrated that prazosin decreases the plasma concentration of inflammatory factors, and improves oxidative stress in myocardial cells (26). Notably, it was observed that prazosin treatment exerts significant anti-apoptotic effects in myocardial cells of mice models of anoxia-reoxygenation injury. These findings indicate that prazosin regulates survival of myocardial cells via the ERK-mediated signaling pathway in a mouse model of anoxia-reoxygenation injury.

Prazosin is a selective postsynaptic al receptor blocker, which is used for treating mild and moderate hypertension (27). Prazosin reduces the load of the heart, which has been used for the treatment of cardiac insufficiency (28). Zhao and $\mathrm{Xu}$ (29) investigated the ameliorative effect of prazosin on diabetic nephropathy patients with positive a1-adrenergic receptor autoantibodies and refractory hypertension. In addition, long-term effects of prazosin treatment on blood pressure, carotid arteries, heart rate, and acetylcholine have been reported in a previous study (30). Furthermore, Qazi et al (31) indicated that prazosin treatment exerts regulatory effects in posttraumatic stress and alcohol use disorders. These reports indicate that prazosin may present as an efficient therapeutic agent for the treatment of anoxia-reoxygenation injury. 
Inflammation is essential in the initiation and development of coronary heart disease caused by anoxia-reoxygenation injury of heart (32). Bobbert et al (33) have suggested that myocardial injury induced by platelet activation and thrombus formation is associated with myocardial inflammation in patients with cardiomyopathy. In addition, the biological importance of inflammatory factor, IL-6-mediated signaling pathways have been elucidated in patients with acute myocardial infarction and dysglycemia (34). Additionally, previous studies indicated that myocardial injury is aggravated by systemic inflammation, which may further induce other types of heart disease $(35,36)$. Furthermore, TNF- $\alpha$ and IL-10 were reported as markers of the inflammatory response in coronary artery disease patients with acute myocardial infarction $(37,38)$. The current results indicate that prazosin is beneficial for improvement of inflammatory factor expression in serum in mice with anoxia-reoxygenation injury. Decreasing serum expression levels of IL-6, TNF- $\alpha$, IL-10 and IL-1 may contribute to upregulation of anti-apoptotic genes expressed in myocardial cells.

Previous studies have indicated that apoptosis of cardiomyocytes is crucial in cardiac dysfunction following acute myocardial infarction in the development of coronary heart disease $(39,40)$. Numerous strategies aimed at preventing or mitigating the extent of apoptosis have been attempted to protect the heart against coronary heart disease-induce apoptosis or death of cardiomyocytes (41-43). In the current study, prazosin treatment significantly inhibited apoptosis of myocardial cells by upregulation of Bcl-2 and p53, as well as downregulation of caspase-3, caspase-9, Bcl-2 and p53 in myocardial cells in a mouse model of anoxia-reoxygenation injury. The results also indicated that the ERK signaling pathway is involved in the protection effects of prazosin-mediated anoxia-reoxygenation injury.

Currently, the molecular mechanisms of treatment for coronary heart disease have been widely investigated (44-46). Research has indicated that upregulation of the MAPK-ERK signaling pathway regulates myocardial ischemia-reperfusion and exerts protective roles in myocardial ischemia-reperfusion injury (47). Furthermore, a previous study demonstrated that activating p-ERK ameliorates acute myocardial infarction in rats by decreasing the expression of inflammatory-associated cytokines (48). In the present study, the activity and expression levels of NF-AT, AP-1 and NF- $\kappa$ B were downregulated in myocardial cells following prazosin treatment. The experiments demonstrate that prazosin-induced upregulation of ERK contributes to survival and growth of myocardial cells, which may attenuate inflammation and oxidative stress in myocardial cells in mice with anoxia-reoxygenation injury.

In conclusion, the current study indicates that prazosin protects myocardial cells against inflammation, oxidative stress and apoptosis by stimulating ERK expression and activity. Thus, prazosin presents as an efficient drug for improving survival of myocardial cells, blood lipid levels, blood pressure, and myocardial function in mice with anoxia-reoxygenation injury. In addition, the findings identify and provide evidence of the cardioprotective advantage of prazosin in animal models of anoxia-reoxygenation injury, and illustrate a potential mechanistic pathway for the beneficial effects of prazosin in cardiovascular disease.

\section{References}

1. Kaligis RW, Adiarto S; Erwinanto 2, Nugroho J, Pradnyana BA, Lefi A and Rifqi S: Carotid intima-media thickness in indonesian subjects with cardiovascular disease risk factors who were not receiving lipid-lowering agents. Int J Angiol 25: 174-180, 2016.

2. Lines SW and Carter AM: Complement and cardiovascular disease-the missing link in haemodialysis patients. Nephron 134: 104, 2016.

3. Seaman CD, George KM, Ragni M and Folsom AR: Association of von Willebrand factor deficiency with prevalent cardiovascular disease and asymptomatic carotid atherosclerosis: The atherosclerosis risk in communities study. Thromb Res 144: 236-238, 2016.

4. Morgia G, Russo GI, Tubaro A, Bortolus R, Randone D, Gabriele P, Trippa F, Zattoni F, Porena M, Mirone V, et al: Prevalence of cardiovascular disease and osteoporosis during androgen deprivation therapy prescription discordant to EAU guidelines: Results from a multi-center cross-sectional analysis from the CHOsIng treatment for prostate canCEr (CHOICE) study. Urology 96: 165-170, 2016.

5. Heikkilä K, Koskinen OA, Agarwal A, Tikkinen KA, Mäki M and Kaukinen K: Associations of coeliac disease with coronary heart disease and cerebrovascular disease: A systematic review and meta-analysis. Nutr Metab Cardiovasc Dis 25: 816-831, 2015.

6. Tully PJ, Turnbull DA, Beltrame J, Horowitz J, Cosh S, Baumeister $\mathrm{H}$ and Wittert GA: Panic disorder and incident coronary heart disease: A systematic review and meta-regression in 1131612 persons and 58111 cardiac events. Psychol Med 45: 2909-2920, 2015.

7. Weinreuter M, Kreusser MM, Beckendorf J, Schreiter FC, Leuschner F, Lehmann LH, Hofmann KP, Rostosky JS, Diemert N, Xu C, et al: CaM Kinase II mediates maladaptive post-infarct remodeling and pro-inflammatory chemoattractant signaling but not acute myocardial ischemia/reperfusion injury. EMBO Mol Med 6: 1231-1245, 2014.

8. Ren-an Q, Juan L, Chuyuan L, Wenjuan F, Chunyan H, Xuemei Y, Lin $\mathrm{H}$ and Hong N: Study of the protective mechanisms of Compound Danshen Tablet (Fufang Danshen Pian) against myocardial ischemia/reperfusion injury via the Akt-eNOS signaling pathway in rats. J Ethnopharmacol 156: 190-198, 2014.

9. Yndestad A, Sandanger $\varnothing$, Jong WMC, Aukrust P and Zuurbier CJ: Response to letter from Toldo et al. on 'NLRP3 inflammasome activation during myocardial ischemia reperfusion is cardioprotective'. Biochem Biophys Res Commun 474: 328-329, 2016.

10. Yang $\mathrm{M}$, Chen $\mathrm{J}$, Zhao J and Meng M: Etanercept attenuates myocardial ischemia/reperfusion injury by decreasing inflammation and oxidative stress. PLoS One 9: e108024, 2014.

11. Chao J, Yin H, Yao YY, Shen B, Smith RS Jr and Chao L: Novel role of kallistatin in protection against myocardial ischemia-reperfusion injury by preventing apoptosis and inflammation. Hum Gene Ther 17: 1201-1213, 2006.

12. Vinten-Johansen J, Jiang R, Reeves JG, Mykytenko J, Deneve J and Jobe LJ: Inflammation, proinflammatory mediators and myocardial ischemia-reperfusion Injury. Hematol Oncol Clin North Am 21: 123-145, 2007.

13. Loubele ST, Spek CA, Leenders P, van Oerle R, Aberson HL, Hamulyák K, Ferrell G, Esmon CT, Spronk HM and ten Cate H: Activated protein $\mathrm{C}$ protects against myocardial ischemia/ reperfusion injury via inhibition of apoptosis and inflammation. Arterioscler Thromb Vasc Biol 29: 1087-1092, 2009.

14. Al-Asmari AK, Al-Seif AA, Hassen MA and Abdulmaksood NA: Role of prazosin on cardiovascular manifestations and pulmonary edema following severe scorpion stings in Saudi Arabia. Saudi Med J 29: 299-302, 2008.

15. Vaziri ND: Role of dyslipidemia in impairment of energy metabolism, oxidative stress, inflammation and cardiovascular disease in chronic kidney disease. Clin Exp Nephrol 18: 265-268, 2014.

16. Matsuda $\mathrm{M}$ and Shimomura I: Roles of adiponectin and oxidative stress in obesity-associated metabolic and cardiovascular diseases. Rev Endocr Metab Disord 15: 1-10, 2014.

17. Csanyi G and Miller FJ Jr: Oxidative stress in cardiovascular disease. Int J Mol Sci 15: 6002-6008, 2014.

18. Belló-Klein A, Khaper N, Llesuy S, Vassallo DV and Pantos C: Oxidative stress and antioxidant strategies in cardiovascular disease. Oxid Med Cell Longev 2014: 678741, 2014. 
19. Molinar-Toribio E, Pérez-Jiménez J, Ramos-Romero S, Lluís L, Sánchez-Martos V, Taltavull N, Romeu M, Pazos M, Méndez L, Miranda A, et al: Cardiovascular disease-related parameters and oxidative stress in SHROB rats, a model for metabolic syndrome. PLoS One 9: e104637, 2014.

20. Kristek F and Koprdova R: Long-term effect of prazosin administration on blood pressure, heart and structure of coronary artery of young spontaneously hypertensive rats. J Physiol Pharmacol 62: 295-301, 2011.

21. Wai-Hoe L, Wing-Seng L, Ismail Z and Lay-Harn G: SDS-PAGE-based quantitative assay for screening of kidney stone disease. Biol Proced Online 11: 145-160, 2009.

22. Jong WM, Ten Cate H, Linnenbank AC, de Boer OJ, Reitsma PH, de Winter RJ and Zuurbier CJ: Reduced acute myocardial ischemia-reperfusion injury in IL-6-deficient mice employing a closed-chest model. Inflamm Res 65: 489-499, 2016.

23. Wang Z, Zhang J, Ren T and Dong Z: Targeted metabolomic profiling of cardioprotective effect of Ginkgo biloba L. extract on myocardial ischemia in rats. Phytomedicine 23: 621-631, 2016.

24. Caraballo JC, Borcherding J, Rector M, Hornick E, Stoltz D, Zabner J and Comellas AP: Role of PON in anoxia-reoxygenation injury: A Drosophila melanogaster transgenic model. PLoS One 9: e84434, 2014.

25. Duan JL, Wang JW, Guan Y, Yin Y, Wei G, Cui J, Zhou D, Zhu YR, Quan W, Xi MM and Wen AD: Safflor yellow A protects neonatal rat cardiomyocytes against anoxia/reoxygenation injury in vitro. Acta Pharmacol Sin 34: 487-495, 2013.

26. Khaidakov M, Mercanti F, Wang X, Ding Z, Dai Y, Romeo F, Sawamura T and Mehta JL: Prevention of export of anoxia/reoxygenation injury from ischemic to nonischemic cardiomyocytes via inhibition of endocytosis. Am J Physiol Heart Circ Physiol 306: H1700-H1707, 2014.

27. Hanon O, Giacomino A, Troy S, Bernaud C, Girerd X and Weber S: Efficacy of and tolerance to prolonged release prazosin in patients with hypertension and non-insulin dependent diabetes. Ann Cardiol Angeiol (Paris) 49: 390-396, 2000 (In French).

28. Rudner S and Browne P: Case report: Management of secondary hypertension in a feline with the use of transdermal prazosin. Int J Pharm Compd 14: 488-491, 2010.

29. Zhao LS and Xu CY: Effect of prazosin on diabetic nephropathy patients with positive $\alpha 1$-adrenergic receptor autoantibodies and refractory hypertension. Exp Ther Med 9: 177-182, 2015.

30. Kristek F, Malekova M and Cacanyiova S: Long-term effect of prazosin and losartan administration on blood pressure, heart, carotid artery, and acetylcholine induced dilation of cardiovascular system of young Wistar rats and SHR. Gen Physiol Biophys 32: 235-243, 2013.

31. Qazi H, Wijegunaratne H, Savajiyani R and Koola MM: Naltrexone and prazosin combination for posttraumatic stress disorder and alcohol use disorder. Prim Care Companion CNS Disord 16, 2014.

32. Carmona F, Manso PH, Silveira VS, Cunha FQ, de Castro M and Carlotti AP: Inflammation, myocardial dysfunction, and mortality in children with septic shock: An observational study. Pediatr Cardiol 35: 463-470, 2014.

33. Bobbert P, Weikert U, Schmidt-Lucke C, Skurk C, Meyer A, Steffens D, Schultheiss HP and Rauch U: Platelet activation and thrombus formation relates to the presence of myocardial inflammation in patients with cardiomyopathy. J Cardiol 63: 379-384, 2014.

34. Ritschel VN, Seljeflot I, Arnesen H, Halvorsen S, Weiss T, Eritsland $\mathrm{J}$ and Andersen GØ: IL-6 signalling in patients with acute ST-elevation myocardial infarction. Results Immunol 4: $8-13,2013$.
35. Langhorn R, Persson F, Ablad B, Goddard A, Schoeman JP, Willesen JL, Tarnow I and Kjelgaard-Hansen M: Myocardial injury in dogs with snake envenomation and its relation to systemic inflammation. J Vet Emerg Crit Care (San Antonio) 24: 174-181, 2014.

36. de Gaetano M, Quacquaruccio G, Di Castelnuovo A, Nowak N, Dorn J, Donati MB, Freudenheim JL, Trevisan M and Iacoviello L: Haplotypes and haplotype-pairs of IL-1 beta and IL-6 genes and risk of non fatal myocardial infarction in the Western New York Acute MI Study. Thromb Haemost 106: 1231-1233, 2011.

37. Li X, Luo R, Chen R, Song L, Zhang S, Hua W and Chen H: Cleavage of I $\kappa \mathrm{B} \alpha$ by calpain induces myocardial NF- $\kappa \mathrm{B}$ activation, TNF- $\alpha$ expression and cardiac dysfunction in septic mice. Am J Physiol Heart Circ Physiol 306: H833-H843, 2014.

38. Goswami B, Rajappa M, Mallika V, Shukla DK and Kumar S: TNF-alpha/IL-10 ratio and C-reactive protein as markers of the inflammatory response in CAD-prone North Indian patients with acute myocardial infarction. Clin Chim Acta 408: 14-18, 2009.

39. Hashemian M, Poustchi H, Mohammadi-Nasrabadi F and Hekmatdoost A: Systematic review of zinc biochemical indicators and risk of coronary heart disease. ARYA Atheroscler 11: 357-365, 2015.

40. Farvid MS, Ding M, Pan A, Sun Q, Chiuve SE, Steffen LM, Willett WC and Hu FB: Response to letters regarding article, 'dietary linoleic acid and risk of coronary heart disease: A systematic review and meta-analysis of prospective cohort studies'. Circulation 132: e23-e24, 2015.

41. Liu LL, Lin LR, Lu CX, Fu JG, Chao PL, Jin HW, Zhang ZY and Yang TC: Expression of inflammatory and apoptosis factors following coronary stent implantation in coronary heart disease patients. Int Immunopharmacol 11: 1850-1854, 2011.

42. Salmina AB, Shul'man VA, Nikulina SY, Trufanova LV, Fursov AA, But'yanov PA, Kuskaev AP, Bol'shakova EV and Kotlovskii MY: Apoptosis of leukocytes as a marker of neutrophil-endotheliocyte interaction in coronary heart disease. Bull Exp Biol Med 144: 39-41, 2007 (In English, Russian).

43. Geng YJ: Molecular mechanisms for cardiovascular stem cell apoptosis and growth in the hearts with atherosclerotic coronary disease and ischemic heart failure. Ann N Y Acad Sci 1010: 687-697, 2003.

44. Ren Y, Yang H, Browning C, Thomas S and Liu M: Performance of screening tools in detecting major depressive disorder among patients with coronary heart disease: A systematic review. Med Sci Monit 21: 646-653, 2015.

45. Kim C, Cushman M, Kleindorfer D, Lisabeth L, Redberg RF and Safford MM: A review of the relationships between endogenous sex steroids and incident ischemic stroke and coronary heart disease events. Curr Cardiol Rev 11: 252-260, 2015.

46. Ray IB, Menezes AR, Malur P, Hiltbold AE, Reilly JP and Lavie CJ: Meditation and coronary heart disease: A review of the current clinical evidence. Ochsner J 14: 696-703, 2014.

47. Jiang M, Wang L and Jiang HH: Role of spinal MAPK-ERK signal pathway in myocardial ischemia-reperfusion injury. Zhongguo Dang Dai Er Ke Za Zhi 15: 387-391, 2013 (In Chinese).

48. Duan J, Yang Y, Liu H, Dou PC and Tan SY: Osthole ameliorates acute myocardial infarction in rats by decreasing the expression of inflammatory-related cytokines, diminishing MMP-2 expression and activating p-ERK. Int J Mol Med 37: 207-216, 2016.

This work is licensed under a Creative Commons Attribution-NonCommercial-NoDerivatives 4.0 International (CC BY-NC-ND 4.0) License. 